\title{
BIRATIONAL TRANSFORMATIONS OF CERTAIN QUARTIC
}

\author{
SURFACES*
}

BY

\section{F: R. SHARPE AND VIRGIL SNYDER}

1. Statement of the problem. It has been shown by Enriques $\dagger$ that if an algebraic surface contains a pencil of elliptic curves and also two curves cutting each curve of the pencil in two groups of points which are not equivalent and which do not possess equivalent multiples, the surface is invariant under a discontinuous infinite series of birational transformations. In a more recent memoir $\ddagger$ the same author states that, with the exception of the case in which

$$
p_{o}=p_{a}=P_{2}=p^{(1)}=1,
$$

the converse is also true, since transversal curves of each curve of the pencil can always be found. Since the publication of the second theorem the author has discovered that the conclusion must be modified.§ An interesting example of a quartic surface having an infinite discontinuous group of birational transformations but not containing any elliptic curves has been found by Fano.\| In the following paper it is proposed to study the possibility and the nature of birational transformations of quartic surfaces which pass through one or more given curves. The method of the basis of a system of curves on the surface will be employed, as developed by Severi. $\Upsilon$

- Presented to the Society, February 28, 1914.

†F. Enriques, Sulle superficie algebriche, che ammettono una serie discontinua di trasformazioni birazionali, Rendiconti della Reale Accademia dei Lincei, ser. 5, vol. 15 (1906), pp. 665-669.

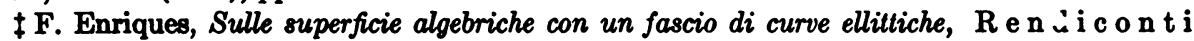
della Reale Accademia dei Lincei, ser. 5, vol. 21 (1912), pp. 14-17.

$\$$ A. Rosenblatt, Sur certaines classes des surfaces algébriques irregulières et sur les transformations birationnelles de ces surfaces en ellesmermes, Bullet in de l'a cadémie des s ci ences de Cracovie, Juillet, 1912, pp. 761-810. 'See footnote 1, page 802.

II G. Fano, Sopra alcune superficie del 4. ordine rappresentabili sul piano doppio, $\mathbf{R}$ e $\mathbf{n}$ dicontidel Reale Istituto Lombardo, ser. 2, vol. 39 (1906), pp. 10711086.

I F. Severi, Sulla totalitd delle curve algebriche tracciate sopra una superficie algebrica, $\mathrm{M}$ at $\mathrm{h}$ e matis che Annalen, vol. 62 (1906), pp. 194-225. 


\section{Quartic Surfaces Containing a Straight Line}

2. General case. If a quartic surface contains a straight line it contains a pencil of plane elliptic cubics. If it is otherwise unrestricted, the surface contains no other rational curves, and consequently no birational transformations into itself.

3. Rational curve on the surface. Let the quartic surface contain the straight line $C_{1}$ and a rational curve $C_{n}$ of order $n$ meeting $C_{1}$ in $n-1$ points. Any plane through $C_{1}$ contains a plane cubic curve $C_{3}$ on $F_{4}$ that meets $C_{n}$ in one point $K$. The involution defined by projecting $C_{3}$ upon itself from $K$ leaves $C_{3}$ invariant. Associated with this involution is a Cremona transformation of the Jonquières type, having the polar conic of $K$ with respect to $C_{3}$ for invariant curve. This Jonquières transformation will be understood when we speak of the involution. As $K$ describes $C_{n}$ the involution defines a Cremona transformation which leaves $F_{4}$ invariant and transforms every $C_{3}$ of the pencil into itself. When the equations of the surface and of $C_{1}$ and $C_{n}$ are known, the equations of the transformation can be readily derived.

The system of curves $C_{1},\left|C_{3}\right|$, and $C_{n}$ constitute a basis, since we are concerned with the most general surface which satisfies the conditions.* The values of $\left[C_{i}, C_{k}\right]$ are

$$
\begin{aligned}
& {\left[C_{1}, C_{1}\right]=-2, \quad\left[C_{3}, C_{3}\right]=0, \quad\left[C_{n}, C_{n}\right]=-2,} \\
& {\left[C_{1}, C_{3}\right]=3, \quad\left[C_{1}, C_{n}\right]=n-1, \quad\left[C_{3}, C_{n}\right]=1 .}
\end{aligned}
$$

In terms of the above curves the transformation may be expressed symbolically as follows.

Let $C_{1}, C_{3}, C_{n}$ go into $\bar{C}_{1}, \bar{C}_{3}, \bar{C}_{n}$ respectively. The equations connecting the old and the new curves are found to be

$$
\bar{C}_{1}=2 C_{1}+2(n+1) C_{3}-3 C_{n},
$$

$T$.

$$
\begin{aligned}
& \bar{C}_{3}=C_{3}, \\
& \bar{C}_{n}=C_{1}+2(n+1) C_{3}-2 C_{n},
\end{aligned}
$$

by the following considerations. To obtain $\bar{C}_{1}$ we determine the image of $C_{1}$ as $K$ describes $C_{n}$. Draw the lines from $K$ to the three points of intersection of $C_{1}$ and the plane cubic curve defined by $C_{1}$ and $K$. As $K$ describes $C_{n}$ these joining lines generate a ruled surface $R$ containing $\bar{C}_{1}$. On this ruled surface $C_{n}$ is a threefold curve, and $C_{1}$ is an $(r-3)$-fold straight line, $r$ being the order of $R$. The intersection of $R$ with $F_{4}$ is a curve of order $4 r$, consisting of $C_{n}$ counted three times, of $C_{1}$ counted $r-2$ times, since the surfaces touch each other at every point of $C_{1}$, and of a residual curve, the

\footnotetext{
* Severi, 1. c.
} 
image of $C_{1}$. The curve $C_{n}$ meets $C_{1}$ in $n-1$ points, corresponding to each one of which $C_{1}$ counts as a double generator of $R$, hence $r-4=2 n-2$. The residual curve therefore has the symbol

$$
2(n+1)\left(C_{3}+C_{1}\right)-2 n C_{1}-3 C_{n}
$$

and is of order $2(n+1) 4-2 n-3 n$.

It is evident that each plane cubic curve $C_{3}$ goes into itself.

The image of $C_{n}$ is the locus of the first tangential of $K$ as $K$ describes $C_{n}$. The tangents to $\left|C_{3}\right|$ at the points of $C_{n}$ describe a ruled surface of order $2(n+1)$ passing through $C_{n}$ and touching $F_{4}$ at each point of $C_{n}$, and having $C_{1}$ as a $(2 n+1)$-fold line. The residual intersection is the required image of $C_{n}$.

From $K$ four tangents can be drawn to $C_{3}$ besides the one at $K$. As $K$ describes $C_{n}$ the four tangents generate a ruled surface $R_{2 n+6}$ of order $2 n+6$, having $C_{n}$ for a fourfold curve and $C_{1}$ for a multiple line of order $2 n+2$, as may be seen as follows: The tangent cone to $F_{4}$ from any point $P$ on $C_{1}$ consists of the tangent plane at $P$ counted twice and of a cone of order 10 on which $C_{1}$ is fourfold. This cone intersects the projecting cone of $C_{n}$ from $P$ in $C_{1}$ counted $4(n-1)$ times and in $6 n+4$ other lines. These $6 n+4$ lines are composed of two groups; those of the first are the $2 n+1$ tangents to $C_{3}$ at points of $C_{n}$, each counted twice, and the remaining lines belong to the second group. The lines of the second group are generators of the ruled surface in question. The residual intersection of $R_{2 n+6}$ with $F_{4}$ is a curve $C$ counted twice that remains invariant under the transformation $T$. It is defined symbolically by the equation

or

$$
2 C=(2 n+6)\left(C_{1}+C_{3}\right)-4 C_{n}-(2 n+2) C_{1},
$$

$$
C=2 C_{1}+(n+3) C_{3}-2 C_{n} \text {. }
$$

As $K$ describes $C_{n}$ its first tangential $K^{\prime}$ describes a rational curve. By projecting each $C_{3}$ from the corresponding $K^{\prime}$ upon itself, another Cremona transformation $T^{\prime}$ is defined. Since by $T^{\prime}$ both $\left|C_{3}\right|$ and $C_{n}$ remain invariant, and since

$$
\left[\bar{C}_{1}, \bar{C}_{1}\right]=-2, \quad\left[\bar{C}_{1}, \bar{C}_{3}\right]=3, \quad\left[\bar{C}_{1}, \bar{C}_{n}\right]=n-1,
$$

we easily obtain the equations

$$
T^{\prime} . \quad \bar{C}_{1}=-C_{1}+2(n+5) C_{3}+6 C_{n}, \quad \bar{C}_{3}=C_{3}, \quad \bar{C}_{n}=C_{n} .
$$

The determinants of $T, T^{\prime}$ are each -1 , and their product is 1 ; the operation $\left(T T^{\prime}\right)$ is not periodic. Thus the surface is invariant under an infinite number of birational transformations. In any plane the operation $\left(T T^{\prime}\right)$ 
can be replaced by the product of two projections of the curve upon itself, the center of one of which may be chosen anywhere on the curve. But the operation does not define a continuous group, since the $C_{3}$ associated with $K$ has not a constant modulus as $K$ describes $C_{n}$. $^{*}$

Moreover, it can be shown by the same method as that employed by Severi $\dagger$ that all the effective transformations that leave $F_{4}$ invariant can be expressed in terms of $T$ and $T^{\prime}$.

4. One double point on the line. Let all the curves $C_{3}$ pass through one fixed point $P$ on $C_{1}$, the other points of intersection of $C_{1}, C_{3}$ being variable.

The surface is evidently invariant under the projection of the surface upon itself from the point $P$; the projection is an involutional Cremona transformation of the Jonquières type. The surface is also invariant under the transformation defined by the first tangential of $P$ as in the preceding case. Surfaces of this type necessarily contain rational curves which cut every plane $C_{3}$ of the pencil in one point apart from the point $P$.

Symbolically, the transformation is expressed as follows. For basis we may choose $C_{1}, C_{3}$ and the point $P$ with the following characteristic

$$
\begin{gathered}
{\left[C_{1}, C_{1}\right]=-2, \quad\left[C_{1}, C_{3}\right]=2, \quad\left[C_{3}, C_{3}\right]=0,} \\
{\left[C_{1}, P\right]=1, \quad\left[C_{3}, P\right]=1, \quad[P, P]=-2 .}
\end{gathered}
$$

The point $P$ is a double point on $F_{4}$. The tangent cone to $F_{4}$ at $P$ is a quadric passing through $C_{1}$; the residual intersection is a rational curve of order 7 which passes through $P$ five times. We have therefore

$$
\left[C_{7}, P\right]=5, \quad\left[C_{7}, C_{1}\right]=1, \quad\left[C_{7}, C_{3}\right]=1, \quad\left[C_{7}, C_{7}\right]=-2,
$$

so that the equations of the projection from $P$ are easily found to be

S.

$$
\overline{C_{1}}=C_{1} \text {, }
$$

$$
\bar{C}_{3}=C_{3} \text {, }
$$

$$
\bar{P}=C_{1}+2 C_{3}-P \text {. }
$$

The equations of the transformation defined by the first tangential are similarly found to be

$$
\begin{array}{ll}
\bar{C}_{1} & =-C_{1}+10 C_{3}+4 P, \\
\bar{C}_{3} & =C_{3}, \\
\bar{P} & =P .
\end{array}
$$

* Picard et Simart, Fonctions algébriques de deux variables indépendentes, vol. 2, pp. 517518.

†Rendicontidel Circolo Matematicodi Palermo, vol. 30 (1911), pp. 265-288.

† For the idea of using a point as part of a basis, see F. Severi, La base minima pour la totalité des courbes tracés sur une surface algébrique, A n a l e d e l'ó col e n o r m a le s u pér i e u re, ser. 3, vol. 25 (1908), pp. 449-468. 
The product $\left(S S^{\prime}\right)$ is not periodic, hence the surface is invariant under a group of infinite order.

5. Line an inflexional tangent. The cases in which two distinct or coincident points of intersection of $C_{8}$ and $C_{1}$ are fixed offer no new peculiarities; similarly for the cases of three distinct fixed points or of two coincident and one distinct fixed points. But the case in which all three points are coincident merits special attention. If $C_{1}$ is an inflexional tangent for every $C_{3}$ passing through it, the point of inflexion must be the same point for every curve of the pencil, as is easily seen by considering the binary cubic whose roots define the point of contact.

An equation of the form

$$
\phi\left(x_{2}: x_{3}\right)+m f\left(x_{2}: x_{3}\right)=0
$$

must be a cube in $x_{2}: x_{3}$ for every value of $m$; this is possible only when $\phi \equiv k f, k$ being a constant.

The equation of the most general quartic surface $\Phi_{4}$ passing through a line $\left(x_{1}=0, x_{4}=0\right)$ and having the property that every plane section (cubic curve) through the line will have a fixed point of inflexion $(0,0,1,0)$ on the line and the line itself for inflexional tangent, is of the form

where

$$
\Phi_{4} \equiv x_{1} F_{3}\left(x_{1}, x_{2}, x_{3}, x_{4}\right)+x_{4} \phi_{3}\left(x_{2}, x_{3}, x_{4}\right)=0 \text {, }
$$

and

$$
F_{3}=\sum_{i=1}^{4} \sum_{k=1}^{4} \sum_{l=1}^{4} a_{i k l} x_{i} x_{k} x_{l}, \quad \phi_{3}=\sum_{i=2}^{4} \sum_{k=2}^{4} \sum_{l=2}^{4} b_{i k l} x_{i} x_{k} x_{l},
$$

$$
a_{223}=a_{233}=a_{333}=b_{223}=b_{233}=b_{333}=0 .
$$

The tangent plane to $F_{4}$ at any point of the line $x_{1}=0, x_{4}=0$, except the point $(0,0,1,0)$, is $a_{222} x_{1}+b_{222} x_{4}=0$, and is a fixed plane throughout the line. It is no restriction to call this plane $x_{1}=0$, so that $b_{222}=0$.

The equation of the surface now has the simpler form

where

$$
x_{1} F_{3}+x_{4}^{2} \phi_{2}=0 \text {, }
$$

$$
\phi_{2} \equiv \sum_{i=2}^{4} \sum_{k=2}^{4} b_{i k} x_{i} x_{k} \text {. }
$$

The point $(0,0,1,0)$ is a biplanar point, the equations of the two tangent planes being

$$
a_{133} x_{1}^{2}+a_{334} x_{1} x_{4}+b_{334} x_{4}^{2}=0 \text {. }
$$

One of these planes may be taken for $x_{4}=0$, by putting $a_{183}=0$. The surface may be mapped on a double plane $\left(x_{3}=0\right)$ by projecting from the double point $(0,0,1,0)$. 
The curve of branch points is the intersection of the cone of tangents from the double point. By arranging the equation of the surface in the form

$$
u_{2} x_{3}^{2}+2 u_{3} x_{3}+u_{4}=0,
$$

where $u_{2}=x_{4}\left(a_{334} x_{1}+b_{334} x_{4}\right), u_{3}=$ quadratic in $x_{1}, x_{4}$,

$$
u_{4}=\text { quadratic in } x_{1}, x_{4}+a_{222} x_{1} x_{2},
$$

the equation of the cone may be written in the form

where

$$
u_{3}^{2}-u_{2} u_{4} \equiv K_{6}=p_{3} x_{2}^{3}+p_{4} x_{2}^{3}+p_{5} x_{2}+p_{6}=0,
$$

$$
p_{3}=a_{222} x_{1} x_{4}\left(a_{334} x_{1}+b_{33} x_{4}\right) \text {. }
$$

If now a curve exists on $F_{4}$ which meets every plane cubic through the line $x_{1}=0, x_{4}=0$, in one point, it must be rational and meet the line in $n-1$ points.

Projected from $(0,0,1,0)$, the curve lies on the cone $q x_{2}-q^{\prime}=0$, in which $q, q^{\prime}$ are binary in $x_{1}, x_{4}$ of orders $n-1, n$ respectively.

Since every generator of the cone meets $\Phi_{4}$ in one other point, the locus of this residual point is another rational curve lying on $\Phi_{4}$. The first necessary condition that $q x_{2}-q^{\prime}=0$ shall meet $\Phi_{4}$ in two distinct curves is that $Q$ shall be a contact curve of $K_{6}$. If this condition is satisfied, the equation obtained by eliminating $x_{2}$ between $K_{6}$ and $Q$ has only double roots, so that

is of the form $L^{2} / q^{3}$.

$$
\frac{p_{3}{q^{3}}^{3}+p_{4} \cdot q^{\prime 2} q+p_{5} q^{\prime} \cdot q^{2}+p_{6} q^{3}}{q^{3}}
$$

These conditions can be satisfied by properly choosing the constants in $q^{\prime}$ and $q$. The second condition is that the cone from $(0,1,0,0)$ through the curve of intersection of $\Phi_{4}$ and $Q$ shall be composite.

By writing the equation of $\Phi_{4}$ in the form

$$
\left(u_{2} x_{2}+u_{3}\right)^{2}=K_{6}
$$

and making it simultaneous with $Q$, we have

$$
q^{3}\left(u_{2} x_{2}+u_{3}\right)^{2}=L^{2}
$$

This will be composite when and only when $q$ is a square, hence a further condition is stated in the following

THEOREM. If a rational curve exists on $\Phi_{4}$ cutting every cubic curve through $x_{1}=0, x_{4}=0$ in one point, it must be of odd order.

If $q$ is a square, it can contain only $n / 2-1$ constants, while $q^{\prime}$ contains $n+2$ constants, and $\frac{3}{2}(n+2)$ relations among the coefficients must be satisfied. 
Since

$$
n+2+\frac{n}{2}-1=\frac{3 n+2}{2}
$$

it follows that there are not enough constants to satisfy the conditions, hence: The general quartic surface through the line $x_{1}=0, x_{4}=0$, having a point of inflexion at $(0,1,0,0)$ and $x_{1}=0, x_{4}=0$ for inflexional tangent on the cubic curve in every plane through the line, does not contain any rational curve which cuts every cubic curve once.

The surface does not have an infinite number of birational transformations under which it is invariant, but only the involution defined by projecting $\Phi_{4}$ upon itself from the point $P$.

6. Inflexional tangent obtained by transformation. More generally, given a surface $F_{m}$ of order $m$, having the line $x_{1}=0, x_{4}=0$ for an $(m-3)$-fold line and a rational curve meeting every plane section through the line once, it is possible to transform the surface into a surface of order $m^{\prime}$ having an $\left(m^{\prime}-3\right)$-fold straight line on it, and having the further property that every plane cubic section through the line will have the line for inflexional tangent.

At any point $P$ of the rational curve the tangent $t$ and any other line $n$ through $P$ can be chosen in terms of which the equation of the cubic may be expressed in the form

$$
l t-n^{2}+\cdots=0, \quad l(p) \neq 0 .
$$

The conic $l t-n^{2}=0$ has three point contact with the cubic at $P$, and meets it in three other points $P_{1}, P_{2}, P_{3}$. By using the triangle $P_{1} P_{2} P_{3}$ for triangle of quadratic inversion, the cubic will go over into a cubic and the conic into a straight line having at the point $P_{1}^{\prime}$ (the image of $P$ ) three point contact with the image cubic. Since a net of conics can be drawn having three point contact with the cubic at $P$, there are two independent parameters, enough to make the image line any line in the plane. The coördinates of $P_{1}, P_{2}, P_{3}$ enter the equations of transformation symmetrically, and all the operations can be expressed rationally.

But it has been seen that, if every $C_{3}$ has $C_{1}$ for inflexional tangent, the point of contact of $C_{3}$ with $C_{1}$ is fixed.

It is thus shown that a surface containing a pencil of plane cubic curves and a rational curve cutting each curve of the pencil once can be transformed into another having the given line for inflexional tangent of every plane cubic section through it. The converse, however, is not true. In the transformed surface sufficient restrictions are imposed on the coefficients to permit the existence of a rational curve, and hence of an infinite number. These curves are the transforms of the locus of the successive tangentials of $P$ on the original surface. 


\section{Quartic Surfaces Containing a Conic}

7. $F_{4}$ with two coplanar conics. If one plane section of a quartic surface is composed of two conics, the number of linearly independent curves on the surface is two.*

Since neither conic is a multiple of the other, the two conics $C_{2}$ and $C_{2}^{\prime}$ form a basis. The surface is regular and of geometric genus 1 , and hence the grade $n$ and the genus $\pi$ of any curve $C$ on the surface satisfy the relation

$$
n=2 \pi-2 \text {. }
$$

The curves $C$ are self-adjoint and of dimensions $\dagger \pi$. In the preceding cases the question of the existence of a birational transformation did not need to be considered, as each transformation could be determined by geometric considerations. In the present case the existence is not so evident, and we shall proceed algebraically.

Since the conics $C_{2}, C_{2}^{\prime}$ are rational and meet in four points, any two curves $\lambda C_{2}+\mu C_{2}^{\prime}, \lambda^{\prime} C_{2}+\mu^{\prime} C_{2}^{\prime}$ meet in $i$ points, defined by

$$
i=-2 \lambda \lambda^{\prime}+4\left(\lambda \mu^{\prime}+\lambda^{\prime} \mu\right)-2 \mu \mu^{\prime},
$$

and the grade $n$ of $\lambda C_{2}+\mu C_{2}^{\prime}$ is given by

$$
n=-2 \lambda^{2}+8 \lambda \mu-2 \mu^{2}
$$

Hence $\lambda C_{2}+\mu C_{2}^{\prime}$, which is of order $2 \lambda+2 \mu$, meets $C_{2}$ in $4 \mu-2 \lambda$ points and meets $C_{2}^{\prime}$ in $4 \lambda-2 \mu$ points. It follows that $\lambda$ and $\mu$ are positive integers and that neither can-exceed twice the other except the cases $\lambda=1, \mu=0$ and $\lambda=0, \mu=1$. We shall now show that $C_{2}, C_{2}^{\prime}$ form a minimum basis $\S$ so that all curves on $F_{1}$ are expressible in the form $\lambda C_{2}+\mu C_{2}^{\prime}$.

Suppose $\Gamma$ and $\Gamma^{\prime}$ constitute a minimum basis. Then

$$
\begin{aligned}
& C_{2}=\alpha \Gamma+\beta \Gamma^{\prime}, \\
& C_{2}^{\prime}=\gamma \Gamma+\delta \Gamma^{\prime},
\end{aligned}
$$

in which $\alpha, \beta, \gamma, \delta$ are integers. If $|\Gamma|$ is of grade $a,\left|\Gamma^{\prime}\right|$ of grade $b$, and any curve $|\Gamma|$ meets $\left|\Gamma^{\prime}\right|$ in $h$ points, the grade of any curve $l \Gamma+m \Gamma^{\prime}$ is

$$
a l^{2}+2 h l m+b m^{2} \text {. }
$$

* Bagnera and de Franchis, Le nombre $\rho$ de M. Picard pour les surfaces hyperelliptiques, Rendicontidel Circolo Matematicodi Palermo, vol. 30 (1911), pp. 185-238. See page 187.

†F. Severi, Complementi alla theoria dolle base per la tolalild delle curve di una superfice algebrica, Rendiconti del circolo matematico di Palermo, vol. 30 (1911), pp. 265-288, p. 276.

†F. Severi, Math emati s che Annalen, loc. cit., see p. 222.

\&. Severi, Rendiconti di Palermo, loc. cit., p. 277. 
Since $\lambda C_{2}+\mu C_{2}^{\prime}$ may be written in the form

$$
(\lambda \alpha+\mu \gamma) \Gamma+(\lambda \beta+\mu \delta) \Gamma^{\prime},
$$

we have, by making this identical with $l \Gamma+m \Gamma^{\prime}$,

$a(\alpha \lambda+\gamma \mu)^{2}+2 h(\alpha \lambda+\gamma \mu)(\beta \lambda+\delta \mu)+b(\beta \lambda+\delta \mu)^{2}$

Hence

$$
=-2 \lambda^{2}+8 \lambda \mu-2 \mu^{2} \text {. }
$$

$$
\left(a b-h^{2}\right)(\alpha \delta-\beta \gamma)^{2}=-12 .
$$

Since $a, b$, and $h$ are integers, it follows that $\alpha \delta-\beta \gamma= \pm 1$ or \pm 2 . In the former case $C_{2}$ and $C_{2}^{\prime}$ also form a minimum basis. In any case $a$ and $b$ are even, hence the value \pm 2 is impossible, since it requires $h^{2}$ to be of the form $4 p+3$, in which $p$ is an integer. We have therefore proved that the conics $C_{2}$ and $C_{2}^{\prime}$ form a minimum basis. The following table of possible curves on $F_{4}$ can now be found.

$\begin{array}{cccc}\lambda \text { or } \mu & \mu \text { or } \lambda & \text { Order } & \text { Genus } \\ 1 & 0 & 2 & 0 \\ 1 & 1 & 4 & 3 \\ 2 & 1 & 6 & 4 \\ 2 & 2 & 8 & 9\end{array}$

The curves $\lambda C_{2}+\mu C_{2}^{\prime}, \lambda \geqq \mu$, are the residual intersections of $F_{4}$ with surfaces of order $\lambda$ having $C_{2}^{\prime}$ for a $(\lambda-\mu)$-fold curve. All the formulas can be readily verified by the Cayley-Noether equivalence and postulation theorems.* Hence the conics $C_{2}$ and $C_{2}^{\prime}$ are the only rational curves on the surface; the curves of genus two are composed of the partial series of tangent planes; the only curves of genus three are the plane sections $\left|C_{2}+C_{2}^{\prime}\right|$, etc.

If a birational transformation exists that leaves $F_{4}$ invariant, every linear system of curves is transformed into another linear system of the same grade, genus, and dimension. Hence the plane sections must be transformed into plane sections and the two conics either interchanged or left invariant. This is possible only under a linear transformation, and the general quartic surface containing a conic does not admit such a transformation. We may therefore conclude: The most general quartic surface subjected only to the condition that it must contain a conic is not invariant under any birational transformation. $\dagger$

* M. Noether, Sulle curve multiple di superficie algebriche, A n n a li d i m a t e m a t i c a, ser. 2, vol. 5 (1871), pp. 163-177. See p. 164 and 168.

$\dagger \mathrm{L}$. Godeaux, Sur la surface du quatrième ordre contenant une conique, S o c iét té des s c i e n c e s d a $\mathrm{H}$ a in a u t, vol. 62 (1912), pp. 1-8, has considered the problem of possible birational transformations of the quartic surface containing a conic. In consequence of the restriction $\lambda \ngtr 2 \mu$ (in our notation) which he overlooks, the system of rational curves there found are all composite. The proof of the non-existence of involutional transformations is also incomplete because not all possible cases are considered. 


\section{Quartics Containing One or Two Space Cubic Curves}

8. $F_{4}$ through a space cubic curve. Let $F_{4}$ contain a space cubic curve $C_{3}$. From any point $P$ on $F_{4}$ can be drawn one bisecant to $C_{3}$ which meets $F_{4}$ in a fourth point $P^{\prime}$. Since the bisecant to $C_{3}$ from $P^{\prime}$ meets $F_{4}$ in $P$, we see that $F_{4}$ admits an involutional birational transformation. Through $C_{3}$ pass a bundle of quadric surfaces, each meeting $F_{4}$ in a residual quintic of genus 2. All the quadrics of the bundle which pass through a fixed point $P$ belong to a pencil whose basis curve consists of $C_{3}$ and the bisecant through $P$. Hence all the quintic curves through $P$ on $F_{4}$ also pass through $P^{\prime}$.

A basis is composed of $C_{3}$ and a plane section $C_{4}$. By proceeding as in the preceding cases we obtain the transformation

$U$.

$$
\bar{C}_{4}=9 C_{4}-5 C_{3},
$$$$
\bar{C}_{3}=16 C_{4}-9 C_{8} \text {, }
$$

which can be verified as follows:

The image of a plane section $C_{4}$ is obtained by constructing the ruled surface of bisecants to $C_{3}$ from each point of $C_{4}$. The curve $C_{4}$ is a simple curve on the surface, and the lines joining the three points of intersection of $C_{3}$ and $C_{4}$ in pairs are double generators. Hence the surface is of order 10. From any point of $C_{3}$ a quadric cone of lines can be drawn meeting $C_{3}$ again. This cone meets the quartic cone to $C_{4}$ in eight lines, but three of them pass through the points of intersection of $C_{3}$ and $C_{4}$; hence $C_{3}$ is a five-fold curve on the ruled surface. Since $\bar{C}_{4}$ is the residual intersection of $F_{4}$ and the ruled surface, we have

$$
\bar{C}_{4}=10 C_{4}-\left(C_{4}+5 C_{3}\right) .
$$

The image of $C_{3}$ is obtained as the residual intersection with $F_{4}$ of the ruled surface obtained by connecting each point $K$ of $C_{3}$ with the point $K^{\prime}$ in which the tangent plane to $F_{4}$ at $K$ meets $C_{3}$ again.

From $K$ can be drawn one generator to $K^{\prime}$; from $K^{\prime}$ can be drawn six generators to points of tangency $K$. Moreover, the ruled surface and $F_{4}$ touch each other at every point $K$ of $C_{3}$; hence each generator meets 14 others, and the surface is of order 16 . The curve $C_{3}$ counts for nine intersections, and hence the residual intersection $C_{3}$ is defined by the formula

$$
\bar{C}_{3}=16 C_{4}-9 C_{3} \text {. }
$$

The other possible values of $\lambda$ and $\mu$ which satisfy the congruences analogous to those of $\S 2$ lead to non-effective curves. Hence the surface does not admit an infinite number of birational transformations.

The transformation is a Cremona transformation, the invariant surface 
being the locus of the double points of the involution determined on $C_{3}$ and $F_{4}$ by the bisecants of $C_{3}$.

9. $F_{4}$ through two space cubic curves. Consider the general quartic surface containing the two space cubic curves $C_{3}$ and $C_{3}^{\prime}$ which intersect in $k$ points $0 \leqq k \leqq 5$. A basis may be made of $C_{3}, C_{3}^{\prime}$ and a plane section $C_{4}$. The involution defined by transforming $P$ into the residual point $P^{\prime}$ of intersection with the surface of the bisecant of $C_{3}$ through $P$ may be expressed by the equations

$$
\text { V. } \quad \begin{aligned}
& \bar{C}_{3}=16 C_{4}-9 C_{3}, \\
& \bar{C}_{3}^{\prime}=(12-2 k) C_{4}-(6-k) C_{3}-C_{3}^{\prime}, \\
& \bar{C}_{4}=9 C_{4}-5 C_{3} .
\end{aligned}
$$

The similar involution as to $C_{3}^{\prime}$ has the equations

$$
\begin{array}{ll}
V^{\prime} . & \bar{C}_{3}=(12-2 k) C_{4}-C_{3}-(6-k) C_{3}^{\prime}, \\
& \bar{C}_{3}^{\prime}=16 C_{4}-9 C_{3}^{\prime}, \\
& \bar{C}_{4}=9 C_{4}-5 C_{3}^{\prime} .
\end{array}
$$

The product of these two involutions is a discontinuous birational transformation that is not periodic.

When $k=5$, the curves $C_{3}$ and $C_{3}^{\prime}$ lie on a quadric which meets $F_{4}$ in a conic $C_{2}$. By the transformation $V$ the curves $C_{3}^{\prime}$ and $C_{2}$ are interchanged.

Similarly, the curves $C_{3}$ and $C_{2}$ are interchanged by the transformation $V^{\prime}$.

CORNELL UNIVERSITY, January, 1914. 